\title{
Effects of tilt angle of mirror-lamp system on shape of solid-liquid interface of silicon melt during floating zone growth using infrared convergent heating
}

Md. Mukter Hossain ${ }^{\mathrm{a}}$, Satoshi Watauchia, b, *, Masanori Nagao ${ }^{\mathrm{a}}$, Isao Tanaka ${ }^{\mathrm{a}}$

${ }^{a}$ Center for Crystal Science and Technology, University of Yamanashi, 7-32

Miyamae, Kofu, Yamanashi 400-8511, Japan

'Precursory Research for Embryonic Science and Technology (PRESTO), Japan Science and Technology Agency (JST), 4-1-8 Honcho, Kawaguchi, Saitama 332-0012, Japan *E-mail: watauchi@yamanashi.ac.jp 


\section{Abstract}

The tilt effects of the mirror-lamp (M-L) system on the shape of the interface of the silicon molten zone formed during growth using the infrared convergent heating floating zone method were studied at various positions of the M-L system. The stability and the interfaces of the molten zone formed in the tilted condition were compared with those in the no tilt condition. The molten zone appeared to be more stabilized in the tilted condition than in the no tilt condition. However, the conventional parameters characterizing the interface shape such as convexities $(h / r)$, gap and zone length $(L)$ were almost independent of the tilt angle $(\theta)$ of the M-L system and insufficient to discuss the tilting effects on the molten zone shape. The curvature of the solid-liquid interface was affected by the $\theta$. New characterizing parameters such as the growth interface and triple point angles ( $\delta$ and TPA, respectively) were effective to quantitatively describe the tilting effects on the interface shape. With increase of the $\theta$, the $\delta$ was decreased and the TPA was increased in both the feed and crystal sides. A silicon crystal of $45 \mathrm{~mm}$ in diameter was grown successfully in the tilted condition.

Keywords: A1. Heat transfer, A1. Interface, A2. Floating zone technique, A2. Growth from melt, B2. Semiconducting silicon 


\section{Introduction}

Silicon single crystals are mainly manufactured by both the Czochralski (CZ) and the radio-frequency heating floating zone (rf-FZ) methods [1]. The infrared convergent heating floating zone (IR-FZ) method can also be used to grow silicon single crystals from polycrystalline feed rods [2,3]. However, the IR-FZ method is not applied for the growth of silicon crystals on an industrial scale. The advantages and disadvantages of the IR-FZ method over the $\mathrm{CZ}$ and $\mathrm{rf}-\mathrm{FZ}$ methods are described in our previous reports $[4,5]$. The limited diameter of the grown crystal is one of the main disadvantages of this method. The maximum diameter of a silicon single crystal grown by the IR-FZ method is $\sim 15 \mathrm{~mm} \phi$ [2], which is much smaller than those grown by the $\mathrm{CZ}$ and $\mathrm{rf}-\mathrm{FZ}$ methods $[1]$.

For successful growth using the IR-FZ method, it is very important to maintain a stable molten zone. Contact between the feed rod and grown crystal, and the heat distribution in the molten zone, feed rod, and grown crystal can result in instability of the molten zone even if the molten zone is skillfully controlled [5]. Occasionally, separation of the molten zone occurs when the molten zone is unstable. It is reported that the heat flow and interfaces are significantly related to the stability of the melt [6-8]. This indicates that careful observation and control of the shape of the interface are 
necessary to achieve a more stabilized molten zone because the instability of the molten zone usually limits the diameter of the grown crystal.

During the IR-FZ growth of rutile, the shape of the solid-liquid interface and the stability of the molten zone were significantly affected by the tilt angles $(\theta)$ of the ellipsoidal mirrors during convergent heating. By adjusting the $\theta$ of the mirrors, the decreasing convexity of the shape of the crystal-melt interface and the enhancement of the stability of the molten zone [7,9] were found to be very important factors for the improvement of the diameter and the quality of crystals grown using this method [6]. The shape of the interface also significantly affects the density of crystal defects such as dislocations, and even the presence of inclusions and cracks within the crystal $[9,10]$.

Therefore, in the present study, the effect of the $\theta$ of the mirror-lamp (M-L) system on the shape of the interface of the silicon molten zone during IR-FZ growth was carefully examined. The effect of the position of the tilted $\mathrm{M}-\mathrm{L}$ system was also examined because silicon crystals with a larger diameter were obtained in previous work by changing the position of the $\mathrm{M}-\mathrm{L}$ system during crystal growth using the IRFZ method [5]. The dependencies of the position and the $\theta$ on the parameters that characterize the interface of the molten zone will be discussed. 


\section{Experimental method}

An image furnace (Crystal Systems Corporation: model FZ-T-10000-H-TY-1) with four ellipsoidal mirrors was modified to tilt the mirror at different positions in the $\mathrm{M}-\mathrm{L}$ system. A schematic illustration of this furnace is shown in Fig. 1. The upper parts of each ellipsoidal mirror denoted by dashed lines in Fig. 1, were trimmed so that the mirrors could be tilted. In the furnace, the maximum $\theta$ was set at $10^{\circ}$. A detailed description of the concepts of both the positioning of the M-L system [4,5] and the tilting of the mirrors [9] was provided in previous paper.

Four halogen lamps (each with a maximum power of $1.5 \mathrm{~kW}$ ) were used as a radiation source for the growth of the silicon crystal. A cylindrical polycrystalline silicon $\operatorname{rod}(>11 \mathrm{~N})$ of $15 \mathrm{~mm}$ in diameter was used as a feed crystal. A polycrystalline silicon rod of $20 \mathrm{~mm}$ in diameter was also used as a seed crystal to study the shape of the interface and the stability of the molten zone in these experiments. The feed and seed crystals were positioned on the upper and lower shafts along the same vertical axis, respectively. In all the crystal growth experiments, the following growth parameters were generally used: tilt angle of $10^{\circ}$, growth rate of $5 \mathrm{~mm} / \mathrm{h}$, upper shaft rotation rate of $4 \mathrm{rpm}$, and lower shaft rotation rate of $50 \mathrm{rpm}$. In each growth experiment, the feeding rate was gradually increased from 5 to $10 \mathrm{~mm} / \mathrm{h}$. 
In our present experiments, the mirrors were tilted by an angle of $10^{\circ}$ to investigate the tilting effects on the interface shape quantitatively to compare with the no-tilt $\left(0^{\circ}\right)$ results [4]. Furthermore, the position of the M-L system was also shifted from - $4 \mathrm{~mm}$ to $+4 \mathrm{~mm}$ to demonstrate the position dependence of the $10^{\circ}$ tilted M-L system on the shape of the interface. The system position of - $4 \mathrm{~mm}$ was the closest position to the melt zone which avoided contact of the ellipsoidal mirrors. A thicker silicon crystal using a thicker feed with cross-section of $30 \times 30 \mathrm{~mm}^{2}$ was also tried to be grown in the both no tilt and the tilted conditions at the M-L position of 4, 6, 8 and $10 \mathrm{~mm}$.

To examine the shape of the interface of the silicon molten zone, both the experimental growth conditions and sample preparation for characterization were similar to those previously reported by us [4]. In every experiment, the crystal growth was continued until the crystal diameter was static. Then the molten zone was quenched. Using an electron probe micro-analyzer (EPMA) (JEOL Ltd.: model JXA-8200), the interface shape was characterized by the distribution of iron in the quenched molten zone.

\section{Results and discussion}

During growth experiment, the stability of the molten zone seemed to be enhanced when the $\mathrm{M}-\mathrm{L}$ system was in both the tilted condition and a more distant position. Some 
systematic changes of the shape of the interface could be observed in both the $\theta$ and the position. To discuss the stability of the molten zone quantitatively, several conventional parameters characterizing the shape of the interfaces such as the gap, zone length $(L)$, minimal melt width $\left(w_{\min }\right)$, and height of the interface $\left(h_{\mathrm{c}}, h_{\mathrm{F}}\right)$ can be defined, as shown schematically in Fig. 2(a). Fig. 2(b) shows the iron distributed EPMA mapping images in the quenched silicon molten zone at conventional position $(0 \mathrm{~mm})$ of the $\mathrm{M}-\mathrm{L}$ system with a tilt angle of $0[4]$ and $10^{\circ}$. The shape of the solid-liquid interface could be clearly observed because the segregation coefficient of iron into silicon $\left(\sim 8 \times 10^{-6}\right)$ is much lower than unity. The feed rod and grown crystal are also shown in Fig. 2(b). The diameter of grown crystal was $\sim 20 \mathrm{~mm} \phi$.

As shown in Figs. 2(c), the observed gap values were below $\sim 1 \mathrm{~mm}$. As we mentioned in the previous report [4], the significant effects of the lamp power on the shape of the molten zone have to be excluded. The minimal gap scenario permits the exclusion of these effects. The observed gap values indicate that both experiments were performed with the minimal gap scenario. Therefore, the effects of the $\theta$ of the $\mathrm{M}-\mathrm{L}$ system on the silicon molten zone can be discussed. Figs. 2 (c) and (d) show the effects of the $\theta$ of the $\mathrm{M}-\mathrm{L}$ system on the conventional parameters such as gap, $L, w_{\min }$ and convexities $(h / r)$ at both the feed-melt and crystal-melt sides. These parameters were 
independent of the $\theta$ of the $\mathrm{M}-\mathrm{L}$ system. These results suggest that the conventional parameters are insufficient to discuss the stability of the silicon molten zone. Therefore, new parameters were introduced to characterize the difference in curvature, as shown in Fig. 3(a). These were the growth interface angle $(\delta)$, the triple point angle (TPA), the meniscus angle (MA), and the altitude of the interface curvature (a). Fig. 3(b) shows the magnified EPMA images of the quenched silicon molten zone at the crystal-melt interface region for both the non-tilted $\left(0^{\circ}\right)$ and tilted $\left(10^{\circ}\right)$ conditions at the $0 \mathrm{~mm}$ position of the M-L system. The curvature of the interface was found to be affected by the $\theta$ of the M-L system, even though the $h / r$ defined in Fig. 2(a) is similar.

The values of $a_{\mathrm{F}}$ and $a_{\mathrm{C}}$, which correspond to the altitudes of the feed-melt and the crystal-melt interfaces, respectively, decrease under the $10^{\circ}$ tilt condition, as shown in Fig. 3(c) and (d). It is noted in Figs. 3(c) and (d) that the $\delta$ value decreased and the TPA value increased for both the feed-melt and crystal-melt interfaces under the $10^{\circ}$ tilt condition compared with those of the $0^{\circ}$ tilt condition. The MAF for feed-melt interface shape slightly increased with the increase of the $\theta$. Note here that by careful observation, $w_{\min }$ shown in Fig. 2(c) also found to be slightly increased with the increase of the $\theta$. The behavior of MAF was consistent with that of $w_{\min }$. On the other hand, the MAc for crystal-melt interface was almost independent of the $\theta$. The decrease of $\delta$ and $a_{\mathrm{c}}$ could 
be reduced the possibility of melt drop. Therefore, these can simply be related to the enhancement of the stability of the molten zone.

Figs. 4(a)-(d) show the EPMA mapping images of the iron distribution in the quenched silicon molten zone at different $\mathrm{M}-\mathrm{L}$ system positions with a tilt angle of $10^{\circ}$. The enhancement of the stability of the molten zone with the increase in the position of the $\mathrm{M}-\mathrm{L}$ system was recognized through our experiments. All of the gap values in Fig. 5(a) were also less than $\sim 1 \mathrm{~mm}$. These results also suggest that all the experiments were performed in minimum gap scenario. Therefore, the lamp power effects on the interface shape are excluded in these results. However, not only all the conventional characteristic parameters such as the $L, w_{\min }$, and $h / r$ at both the feed-melt and crystalmelt sides as shown in Figs. 5(a) and (b) but also the newly introduced parameters such as the $\delta, \mathrm{TPA}, \mathrm{MA}, a_{\mathrm{C}}$, and $a_{\mathrm{F}}$ are almost independent of the position of the $\mathrm{M}-\mathrm{L}$ system as shown in Figs. 6(a-h). This implies that the enhancement of the stability of the molten zone with the increase in the position of the M-L system cannot be explained by the behavior of all these parameters. However, the behaviors of these new parameters caused by the mirror tilting at the different positions of the M-L system are consistent with those at $0 \mathrm{~mm}$ position as shown in Figs. 3(c) and (d). As shown in Figs. 5, on the other hand, the behaviors of all conventional parameters in the $10^{\circ}$ tilted conditions as a 
function of the position of the $\mathrm{M}-\mathrm{L}$ system, demonstrated in this experiment were similar with those in the non-tilted condition [4].

The conventional parameters of the interface shapes of the $\mathrm{TiO}_{2}$ (rutile) molten zone was significantly affected by the tilting of the mirror [9] even though the experimental growth conditions for the rutile and silicon crystals were similar, with the exception of the feeding rate and quenching process. The stability of the molten zone was also enhanced as the lamp power and tilt angle increased [9].

The relationship between the stability of the molten zone and the behavior of the convexity was discussed in a previous paper. However, there is a possibility that the obtained shapes of the interfaces of the rutile crystals could differ from the actual shape of the interface. This is because a time of $2-3 \mathrm{~s}$ was required to turn off the lamps after stopping the rotation of both the upper and lower shafts in order to safely obtain the solidified molten zone [6]. In the absence of rotation, forced convection can be replaced by buoyancy-driven convection [11]. The shapes of the interface observed in the EPMA mapping images may be affected by these effects. Under the tilted condition at least, the minimal gap scenario was not clearly recognized in the EPMA mapping images of the quenched rutile melt.

However, the silicon molten zone was quenched very quickly by turning off the 
lamps immediately after stopping the rotation of both the upper and lower shafts. Therefore, the shape of the interface obtained by the iron distribution mapping image could be expected to be more accurate; although theoretically, the shape of the interface is influenced by many parameters such as the heat flow and heat absorption in the molten zone, feed rod, and growing crystal. However, the minimal gap scenario could be recognized in all the EPMA mapping images of the silicon melt, as shown in Figs. 2 and 4.

It was reported that convection flow performs an important function in defining the shape of the interface of oxide materials $[7,12,13]$. However, in the silicon melt, thermal conduction is more dominant than convective heat transfer because silicon has a relatively low Prandtl number [14,15]. Therefore, convection flow such as forced convection caused by the rotation of feed and grown crystal weakly affects the shape of the interface of the silicon melts. This may be one possible reason why the $h / r$ was not affected by either the mirror tilting or the mirror position [4], although the crystal-melt interface fluctuation is caused by the temperature fluctuation induced by Marangoni flow $[16,17]$.

The dependence of the lamp power required for growth on the position of the system in the minimal gap scenario is shown in Fig. 7. In the same M-L system 
position under a tilt angle of $10^{\circ}$, the lamp power required was $\sim 0.3-0.5 \mathrm{~kW}(\sim 5-$ $8 \%$ ) greater than that in the non-tilted condition. For example, the lamp power required for growth when the system was in the zero-position under the non-tilted condition was $4.6 \mathrm{~kW}$, while the power required under a tilt angle of $10^{\circ}$ was $4.9 \mathrm{~kW}$. The required lamp power increased as the position of the M-L system increased, under both the tilted and non-tilted conditions. In the non-tilted condition, there is minimal lamp power required during silicon growth when the $\mathrm{M}-\mathrm{L}$ system is at its nearest position. The most effective heating was achieved under the above conditions.

To obtain a thicker crystal using a thicker feed rod in the non-tilted condition, however, the more distant position of the M-L system was beneficial in the growth of a cylindrical crystal rather than a spiral crystal [5]. The distribution of the convergent infrared light on the surfaces of the feed, molten zone, and grown crystal is significantly affected by the position of the $\mathrm{M}-\mathrm{L}$ system and by the diameter of the crystal. The stability of the molten zone could be related to the focusing of the heating light mainly on the zone-surface.

As we mentioned before, the stability of the molten zone was enhanced in both tilted condition and a more distant position of M-L system. As shown in Fig. 8, using feed 
rods with cross-section of $30 \times 30 \mathrm{~mm}^{2}$, the trial growth experiments for $45 \mathrm{~mm} \phi$ at 4,6 , 8 and $10 \mathrm{~mm}$ position and $0^{\circ}$ tilted condition were not successful, because the molten zone was unstable, causing the sudden melt drop. The molten zone, however, was very stable at $6 \mathrm{~mm}$ position with $10^{\circ}$ tilted conditions. A cylindrical crystal of $45 \mathrm{~mm} \phi$ was grown successfully there. The required lamp power for the growth was $9.3 \mathrm{~kW}$, which is close to the maximum power supply. Therefore, further improvement of the growth diameter was not possible. These results indicate that the stability of the molten zone is enhanced by the mirror tilting even for the growth of a thicker silicon crystal.

The distribution of the convergent light is significantly affected by the mirror tilting, which is closely related not only to the temperature distribution at the convergent area but also to the dynamics of the heat flow in the molten zone. Therefore, the heat flow in the molten zone for $10^{\circ}$ tilted conditions appeared to be different from that for non-tilted condition due to the variation of the temperature gradient at feed-melt and crystal-melt interfaces in the tilted condition.

The observed tilting effects on the new parameters such as $\delta$, TPA, MA, $a_{\mathrm{F}}$ and $a_{\mathrm{C}}$, and on the diameter of the grown crystal are consistent with the enhancement of the stability of the molten zone which would be attributed to the dynamics of the heat flow in the tilted condition. However, the mechanism of the tilting effects of the M-L system 
on the shape of the molten zone is not clear yet. For further discussion about the stability

of the molten zone, both the experimental and simulation study on the melt convection and the temperature distribution in the molten zone are important.

\section{Conclusions}

The shape of the molten zone during the growth of silicon crystals by the floating zone method using infrared convergent heating was studied. The relationship between the shape of the molten zone and both the tilt angle and position of the M-L system is discussed from the perspective of the stability of the molten zone. Newly introduced parameters including the growth interface angle $(\delta)$, the triple point angle (TPA), and the altitude of the curvature of the interface $\left(a_{\mathrm{F}}, a_{\mathrm{C}}\right)$ of the feed-melt and crystal-melt were determined to be dependent on the tilt angle of the $\mathrm{M}-\mathrm{L}$ system, although the convexities $\left(h_{\mathrm{F}} / r_{\mathrm{F}}, h_{\mathrm{C}} / r_{\mathrm{C}}\right)$ of the feed-melt and crystal-melt interfaces did not change. The behavior of these new parameters appeared to be consistent with the stability of the melt. The diameter of the grown crystal was successfully improved in the tilted condition.

\section{Acknowledgments}


This work was partially supported by the Japan Science and Technology Agency (JST),

Precursory Research for Embryonic Science and Technology (PRESTO). 


\section{References}

[1] W. Zulehner, Mater. Sci. Eng. B 73 (2000) 7-15.

[2] A. Eyer, B.O. Kolbesen, R. Nitsche, J. Cryst. Growth 57 (1982) 145-154.

[3] M.A. Gonik, A. Cröll, CrystEngComm 15 (2013) 2287-2293.

[4] M.M. Hossain, S. Watauchi, M. Nagao, I. Tanaka, CrystEngComm 16 (2014) $4619-4623$.

[5] M.M. Hossain, S. Watauchi, M. Nagao, I. Tanaka, Cryst. Growth Des. 14 (2014) $5117-5121$

[6] S. Watauchi, M. Abdur Razzaque Sarker, M. Nagao, I. Tanaka, T. Watanabe, I. Shindo, J. Cryst. Growth 360 (2012) 105-110.

[7] M. Abdur Razzaque Sarker, S. Watauchi, M. Nagao, T. Watanabe, I. Shindo, I. Tanaka, J. Cryst. Growth 317 (2011) 135-138.

[8] C.W. Lan, J. Cryst. Growth 247 (2003) 597-612.

[9] M. Abdur Razzaque Sarker, S. Watauchi, M. Nagao, T. Watanabe, I. Shindo, I. Tanaka, J. Cryst. Growth 312 (2010) 2008-2011.

[10] K. Kitamura, S. Kimura, S. Hosoya, J. Cryst. Growth 48 (1980) 469-472.

[11] N. Kobayashi, J. Cryst. Growth 66 (1984) 63-67.

[12] M. Higuchi, K. Kodaira, Mater. Res. Bull. 29 (1994) 545-550. 
[13] K. Kitamura, N. II, I. Shindo, S. Kimura, J. Cryst. Growth 46 (1979) 277-285.

[14] N. Kobayashi, W.R. Wilcox, J. Cryst. Growth 59 (1982) 616-624.

[15] C.E. Chang, W.R. Wilcox, Int. J. Heat Mass Transf. 19 (1976) 355-366.

[16] M. Sumiji, S. Nakamura, T. Hibiya, J. Cryst. Growth 235 (2002) 55-59.

[17] M. Sumiji, S. Nakamura, T. Azami, T. Hibiya, J. Cryst. Growth 223 (2001) $503-511$ 


\section{Figure captions}

Fig. 1. Schematic of the tilted mirror-lamp (M-L) system at a more distant position.

The definition of the tilt angle $(\theta)$ and the horizontal shift of the system are provided.

Closed circle indicates the conventional focus point of the $\mathrm{M}-\mathrm{L}$ system; this is defined as the zero position. Open circles indicate the more distant focus positions of the system.

The more distant positions of the $\mathrm{M}-\mathrm{L}$ system are defined to be in the positive $(+)$ directions of the ellipsoidal mirrors.

Fig. 2. Summary of conventional parameters characterizing the shape of the molten zone as a function of the tilt angle of the mirror-lamp (M-L) system, with an illustration of the definitions of the parameters: (a) Illustration showing the definitions of characteristic parameters such as the zone length $(L)$, gap, minimal melt width $\left(w_{\text {min }}\right)$, and convexities $\left(h_{\mathrm{F}} / r_{\mathrm{F}}, h_{\mathrm{C}} / r_{\mathrm{C}}\right)$ of the molten zone, (b) EPMA mapping images of the iron distribution in the quenched melt prepared at tilt angle of 0 [4] and $10^{\circ}$, (c) gap, $L(\mathrm{~mm})$ and $w_{\min }(\mathrm{mm})$, and (d) $h / r$; at zero (0) position are given as a function of the tilt angle $(\theta)$ of the $\mathrm{M}-\mathrm{L}$ system.

Fig. 3. Summary of new parameters characterizing the curvature of the feed-melt and crystal-melt interfaces prepared at a mirror-lamp (M-L) system position of zero $(0) \mathrm{mm}$ :

(a) schematic showing the definition of altitude of the curvature of interface $\left(a_{\mathrm{F}}, a_{\mathrm{c}}\right)$ 
and; growth interface, triple point and meniscus angle ( $\delta$, TPA, and MA, respectively), (b) EPMA mapping images (crystal side) showing the variation in $a_{\mathrm{C}}(\mathrm{mm})$ at tilt angles of 0 and $10^{\circ}$, (c) $\delta$, TPA, MA (deg.) and $a_{\mathrm{F}}(\mathrm{mm})$ for feed side, and (d) $\delta$, TPA, MA (deg.) and $a_{\mathrm{C}}(\mathrm{mm})$ for crystal side; as a function of tilt angle $(\theta)$ of the M-L system.

Fig. 4. Cross-sections of iron-distributed mapping images of quenched silicon melt prepared at different positions of the mirror-lamp (M-L) system with a tilt angle of $10^{\circ}$ (a) -4 , (b) 0 , (c) +2 , and (d) $+4 \mathrm{~mm}$.

Fig. 5. Conventional parameters characterizing the silicon molten zone prepared at a tilt angle of $10^{\circ}$ with different position of the mirror-lamp (M-L) system: (a) zone length $(L)$, gap and minimal melt width $\left(w_{\text {min }}\right)$, and (b) convexity $(h / r)$; as a function of position of the $\mathrm{M}-\mathrm{L}$ system. The vertical dashed lines indicate the conventional position of the M-L system defined as zero (0) position.

Fig. 6. Mirror position dependence of the parameters characterizing the feed-melt and crystal-melt interface shape in both non-tilted and $10^{\circ}$ tilted conditions: here (a) growth interface angle $(\delta),($ b) triple point angle (TPA), (c) meniscus angle (MA), and (d) altitude $\left(a_{\mathrm{F}}\right)$ as functions of M-L system position are shown for feed side, and are shown for crystal side at the crystal-liquid-gas point in (e), (f), (g), and (h) respectively. The triangle up (open) and triangle down (closed) data are for 0 and $10^{\circ}$ tilted conditions, 
respectively. The vertical dashed lines indicate the conventional position of the $\mathrm{M}-\mathrm{L}$ system defined as zero (0) position.

Fig. 7. The lamp power required for growth, as a function of the M-L system position. The vertical dashed line shows the conventional position of the M-L system defined as zero (0) position.

Fig. 8. Summary of trial growth experiment of silicon crystal of 20, 30, 40 [5] and 45 $\mathrm{mm} \phi$ as a function of the position of M-L system. Open circles, closed circles and crossed lines indicate the cylindrical, spiral crystal and melt drop at $0^{\circ}$ tilted condition of the mirror-lamp (M-L) system, respectively. Circle, $\mathrm{x}$-hair indicates cylindrical crystal at an angle of $10^{\circ}$ of the M-L system. The vertical dashed line shows the conventional position of the M-L system defined as zero (0) position. 


\section{Figure}

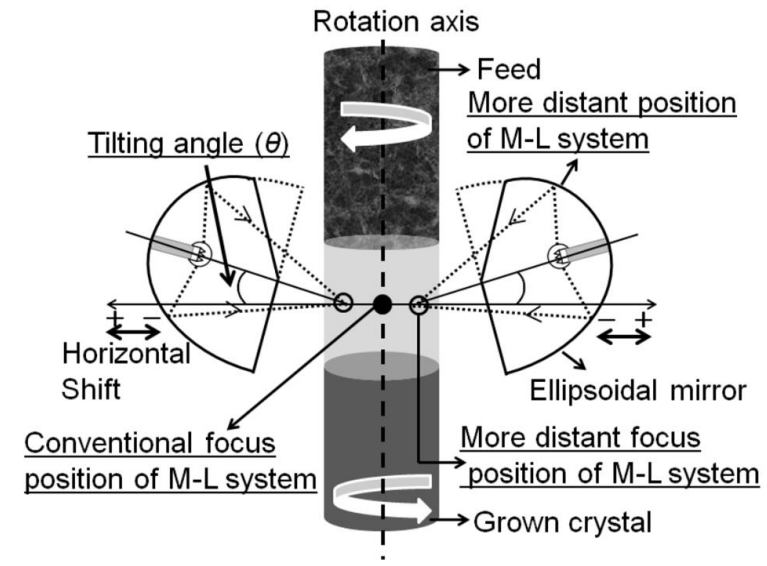

Fig. 1. (Hossain et al.) 


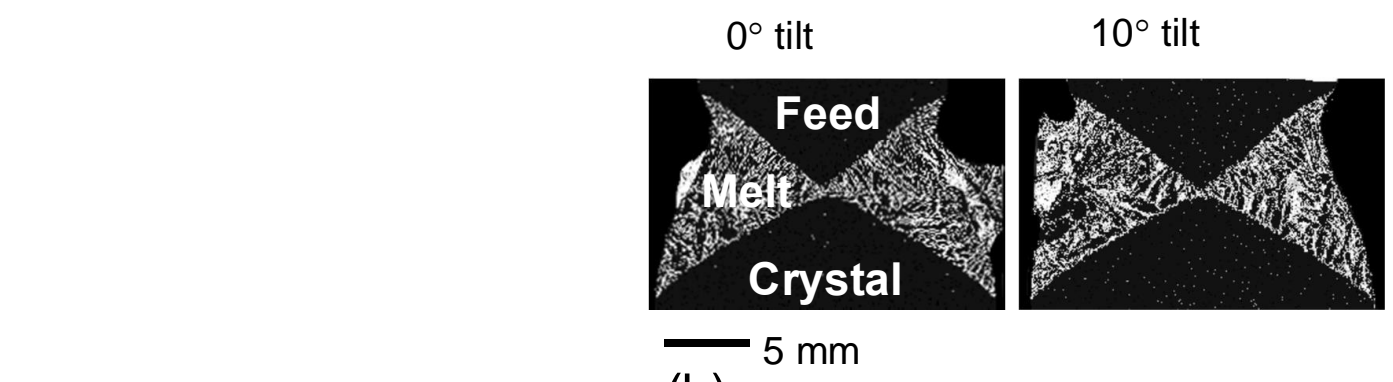

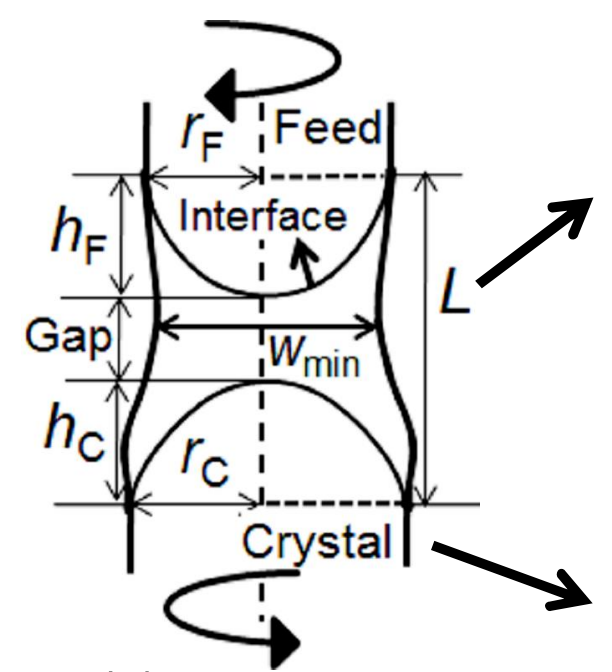

(a)

(b)
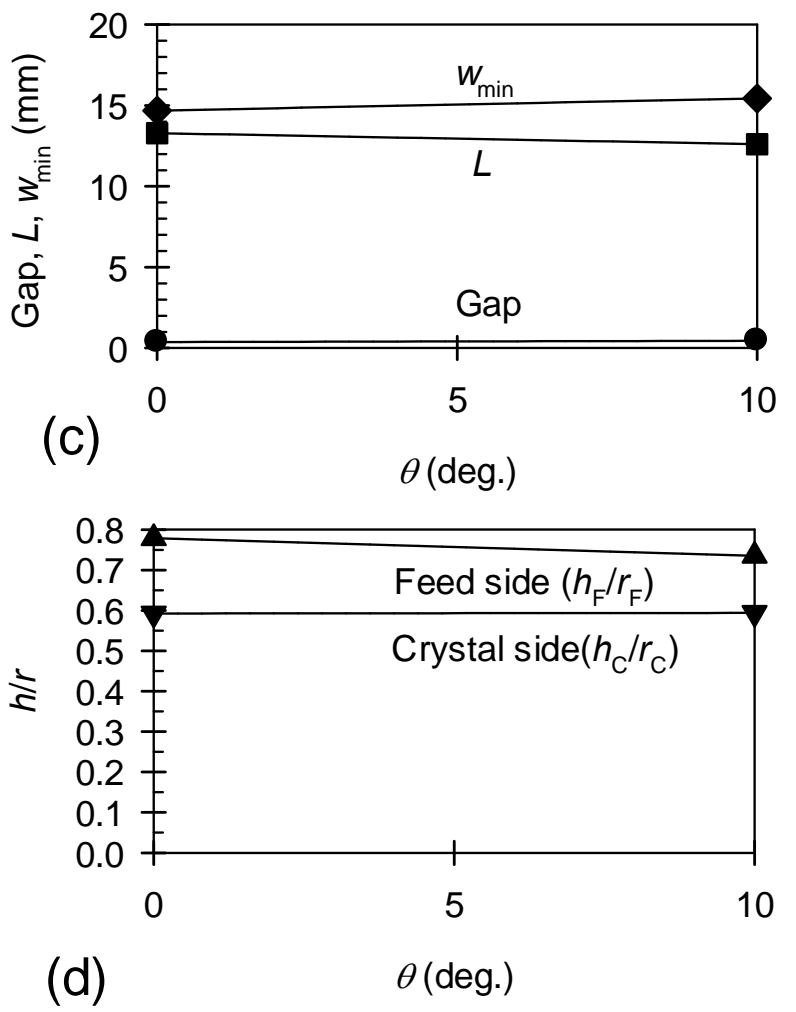

Fig. 2. (Hossain et al.) 


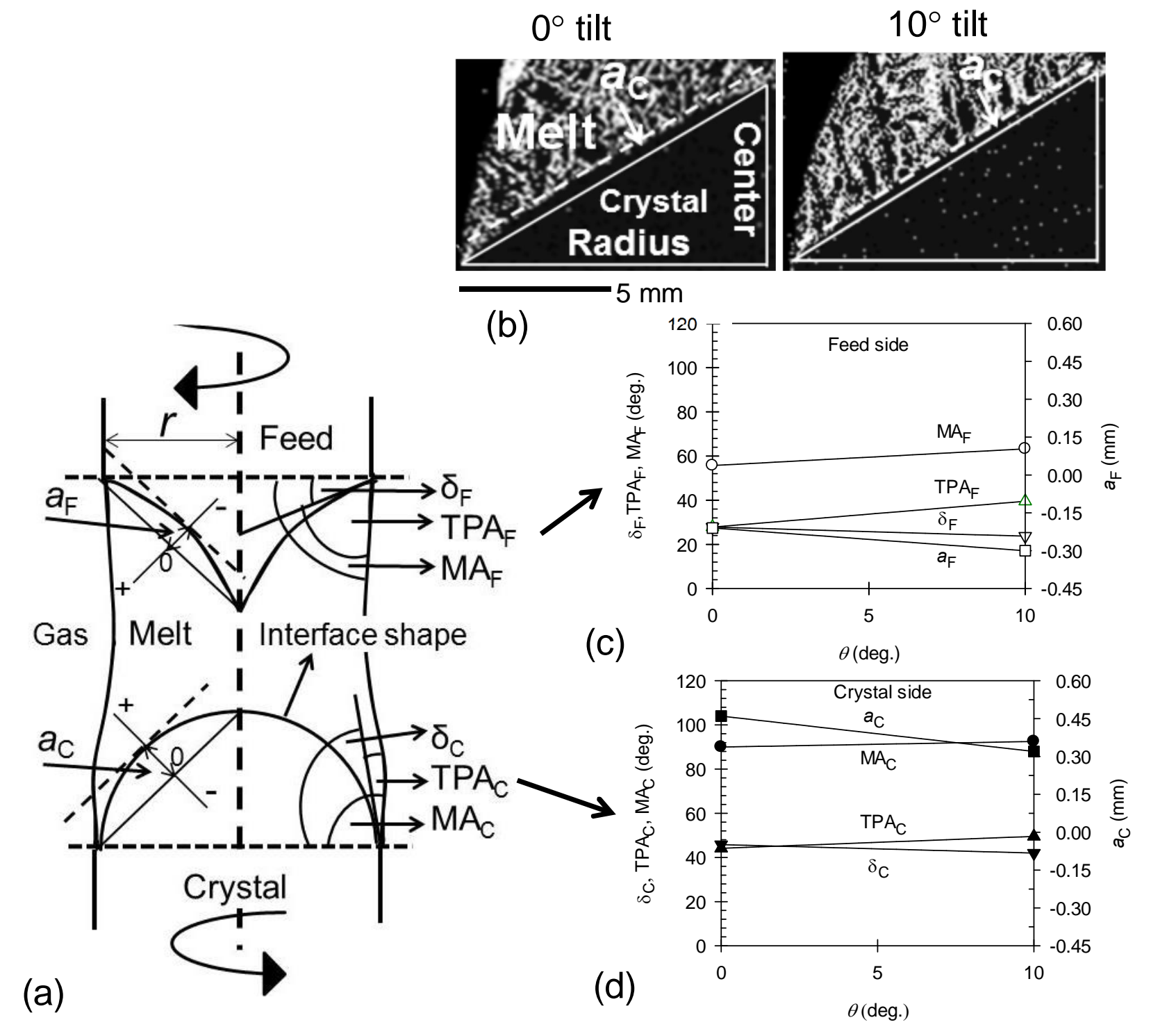

Fig. 3. (Hossain et al.) 

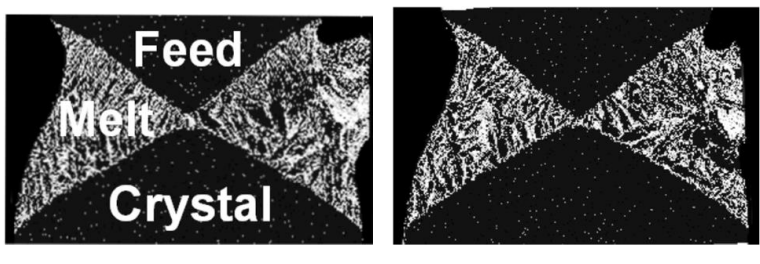

(a)

$5 \mathrm{~mm}$

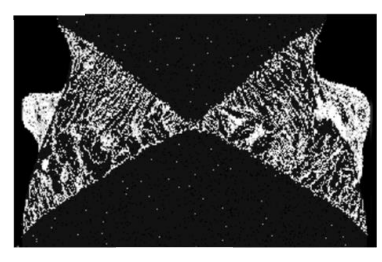

(C) $5 \mathrm{~mm}$

(c)

(b)

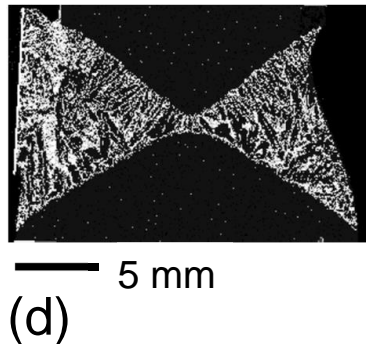

Fig. 4. (Hossain et al.) 

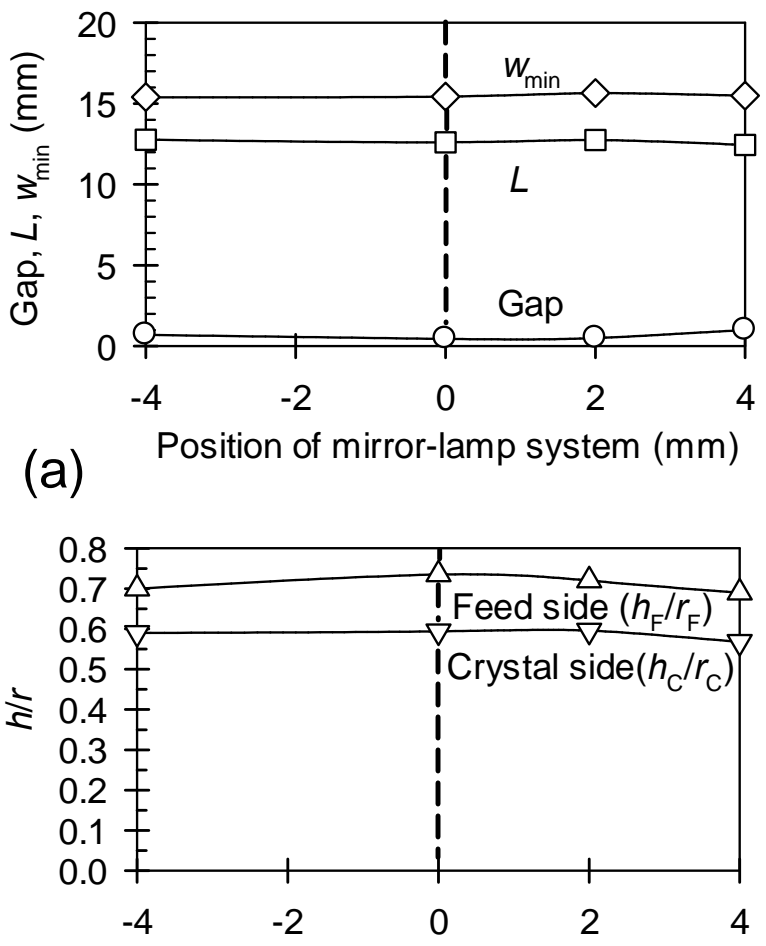

(b) Position of mirror-lamp system $(\mathrm{mm})$

Fig. 5. (Hossain et al.) 

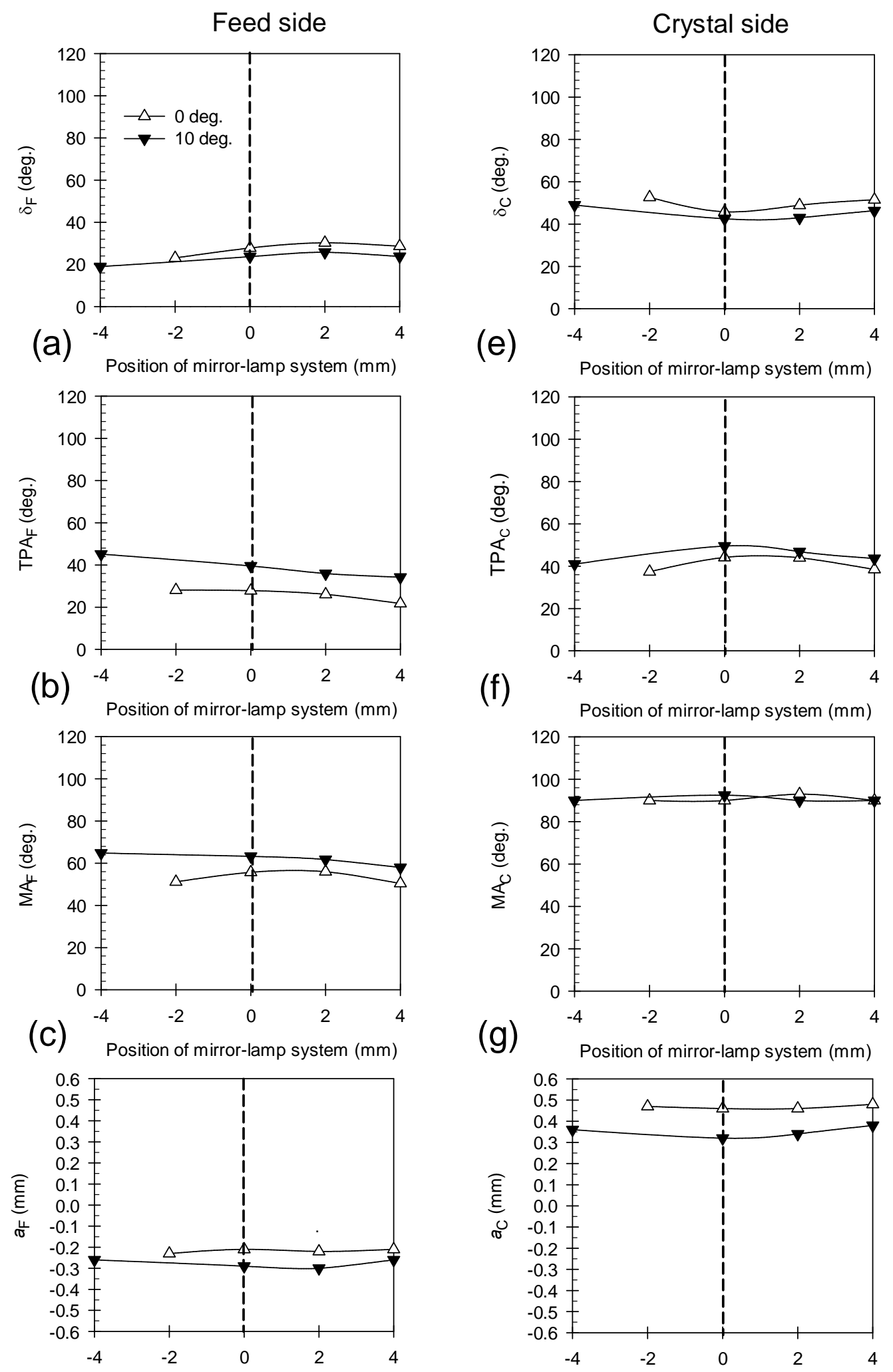

(d) position of mirror-lamp system ( $\mathrm{mm}$ )

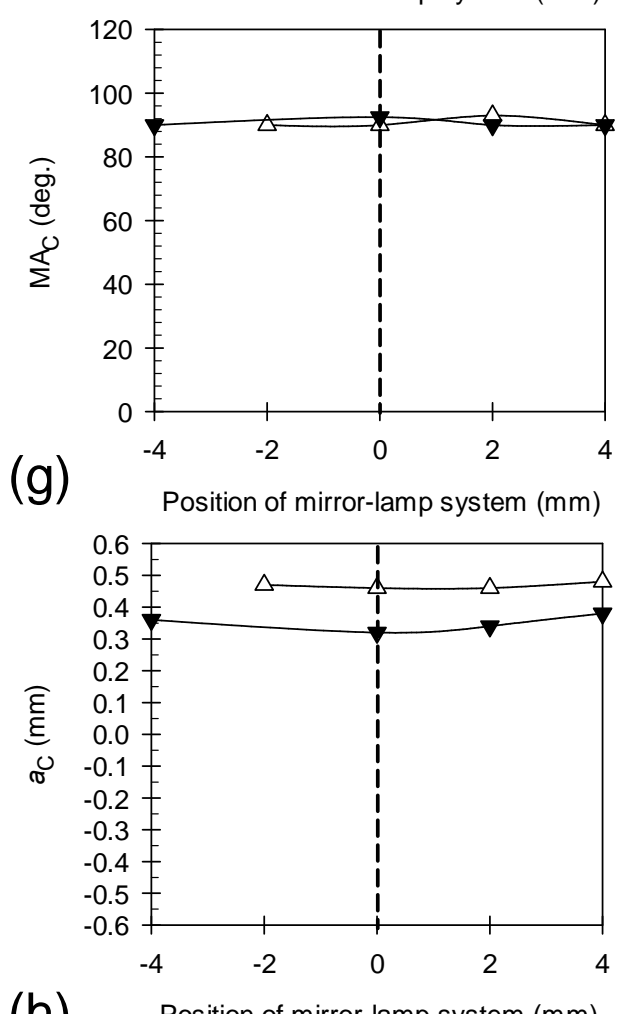

(h) Position of mirror-lamp system ( $\mathrm{mm}$ )

Fig. 6. (Hossain et al.) 


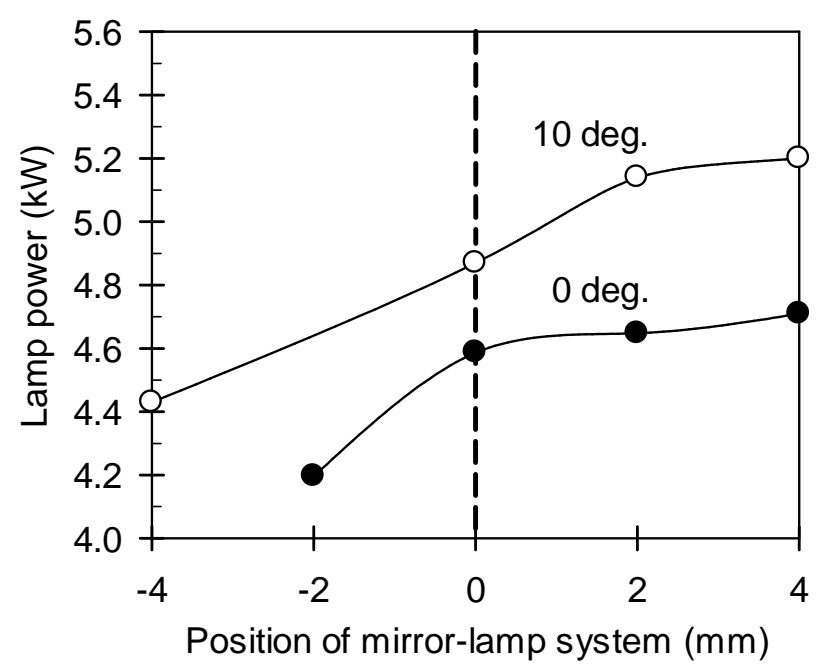

Fig. 7. (Hossain et al.) 


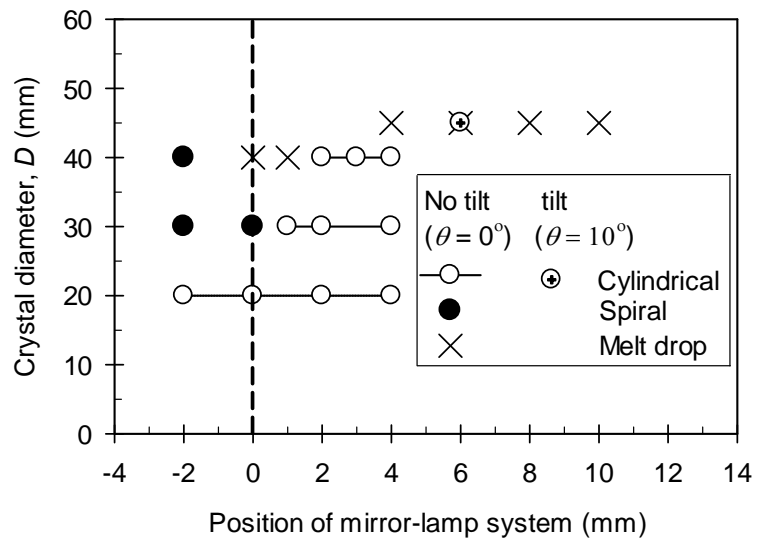

Fig. 8 . (Hossain et al.) 